\title{
Standardization of bilateral upper mediastinal lymph node dissection using microanatomical concepts in minimally invasive esophagectomy
}

\author{
Yasuhiro Shirakawa, Kazuhiro Noma, Naoaki Maeda, Shunsuke Tanabe, Kazufumi Sakurama, Toshiyoshi \\ Fujiwara
}

Department of Gastroenterological Surgery, Okayama University Graduate School of Medicine, Dentistry and Pharmaceutical Sciences, Okayama 700-8558, Japan.

Correspondence to: Dr. Yasuhiro Shirakawa, Department of Gastroenterological Surgery, Okayama University Graduate School of Medicine, Dentistry and Pharmaceutical Sciences, 2-5-1 Shikata-cho, Kita-ku, Okayama 700-8558, Japan.

E-mail: yasuwr@md.okayama-u.ac.jp

\begin{abstract}
How to cite this article: Shirakawa Y, Noma K, Maeda N, Tanabe S, Sakurama K, Fujiwara T. Standardization of bilateral upper mediastinal lymph node dissection using microanatomical concepts in minimally invasive esophagectomy. Mini-invasive Surg 2020;4:33. http://dx.doi.org/10.20517/2574-1225.2020.30
\end{abstract}

Received: 15 Mar 2020 First Decision: 8 Apr 2020 Revised: 15 Apr 2020 Accepted: 24 Apr 2020 Published: 16 Jun 2020

Science Editor: Itasu Ninomiya Copy Editor: Jing-Wen Zhang Production Editor: Jing Yu

\begin{abstract}
Aim: We have recently standardized upper mediastinal lymph node dissection (UMLND) based on microanatomical concepts in minimally invasive esophagectomy using a 4K ultra-high-definition (HD) system. In this study, the aim was to investigate the outcomes of microanatomy-based standardization using 4K ultra-HD for UMLND with the main focus on thoracoscopic operative time.
\end{abstract}

Methods: We have performed more than 500 cases of thoracoscopic esophagectomy in the prone position as minimally invasive esophagectomy. After about 400 cases of thoracoscopic esophagectomy in the prone position, we established the microanatomy-based standardization of UMLND using a 4K ultra-HD system. Two groups were analyzed: a pre-standardization group $(n=100)$ and a post-standardization group $(n=100)$. Furthermore, the change in our thoracoscopic operative time for all cases was analyzed using the moving average method.

Results: In the post-standardization group, the rate of surgeries performed by operators with less than 20 years' experience was significantly higher $(P<0.001)$. There were no significant differences in the number of mediastinal lymph nodes dissected, intraoperative blood loss and total postoperative morbidity rates between the two groups. The rate of recurrent laryngeal nerve palsy decreased to less than half $(19.8 \%$ to $9.6 \%)(P=0.061)$ and the thoracoscopic operative time decreased [232.0 (202.8-264.0) $\mathrm{min}$ to 209.0 (176.0-235.0) min] significantly

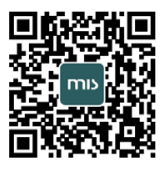


$(P<0.001)$ after standardization. The moving average showed a marked decrease of thoracoscopic operative time during the standardization phase.

Conclusion: Microanatomy-based standardization enabled quicker and more precise UMLD despite an increase in the number of surgeries performed by less experienced operators.

Keywords: Minimally invasive esophagectomy, lymph node dissection, microanatomy, thoracoscopic operative time, recurrent laryngeal nerve palsy

\section{INTRODUCTION}

Esophageal cancer (EC) is one of the most common gastrointestinal malignancies, mainly in Asian countries, and has a poor prognosis ${ }^{[1]}$. Even today, primary treatment is still radical esophagectomy with regional lymphadenectomy ${ }^{[2]}$. The surgical strategy for EC though has been shifting towards minimally invasive esophagectomy (MIE). Currently, thoracoscopic esophagectomy is the most common type of MIE. In 1992, the world's first thoracoscopic esophagectomy was performed in the lateral decubitus position $^{[3]}$. For a period of time after, thoracoscopic esophagectomy in the lateral decubitus position (TELP) was the standard in MIE, and much progress was made, especially in Japan ${ }^{[4,5]}$. Although thoracoscopic esophagectomy in the prone position (TEPP) was reported slightly later than $\operatorname{TELP}^{[6,7]}$, this procedure had not been used for a while. However, in 2006, Palanivelu et al. ${ }^{[8]}$ reported about 130 cases of TEPP and showed both decreased operative time and the frequency of respiratory complications compared with TELP and open esophagectomy. It was also reported that the main reasons for TEPP's usefulness were due to the advantages of good exposure of the surgical field and improved ergonomics for the surgeon. Since then, TEPP has increasingly been adopted all over the world, including here in Japan, and there have been several reports of the tolerability and efficacy of this procedure ${ }^{[9-12]}$.

Upper mediastinal lymph node dissection (UMLND) remains the most important procedure in esophageal cancer surgery. However, this has also been the most difficult and time-consuming part, especially in TEPP. Although there have been technical reports about UMLND in TEPP, the longer thoracoscopic operative times and the higher recurrent laryngeal nerve (RLN) palsy rates of $10 \%$-28\% represent persistent challenges ${ }^{[9,13,14]}$. Recently, progress in the development of endoscopic optical instruments [2K full highdefinition (HD), $4 \mathrm{~K}$ ultra-HD, and 3-dimensional] has been remarkable. Using them, we have been able to identify the fine microanatomy of membranes and layers that were not previously visualized, and there have been some reports on this new concept of surgical microanatomy and its usefulness in esophageal cancer surgery $^{[13-18]}$.

In our institution, we have performed more than 500 cases of TEPP. Since reaching around 350 cases, we have been able to use a $4 \mathrm{~K}$ ultra-HD system for our surgeries. Therefore, we started microanatomy-based standardization of UMLND using this endoscopically magnified view and established it when we reached around 400 cases. Previously, we have reported the concept of this standardization on the left side and its usefulness for safe and efficient surgery, especially for decreasing recurrent laryngeal nerve palsy rates ${ }^{[19]}$. Concurrently, we have also standardized UMLND on the right side with the same concept as on the left within the same period.

The aim of this study was to investigate the outcomes of our microanatomy-based standardized procedure for UMLND on both sides using a $4 \mathrm{~K}$ ultra-HD system, with a focus on decreasing operative time. 
A

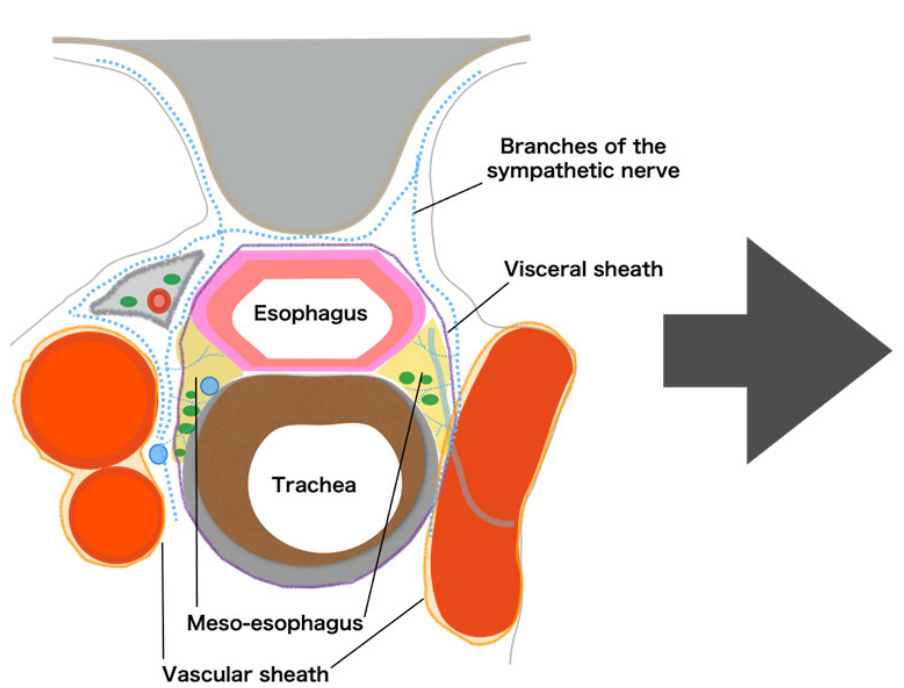

B

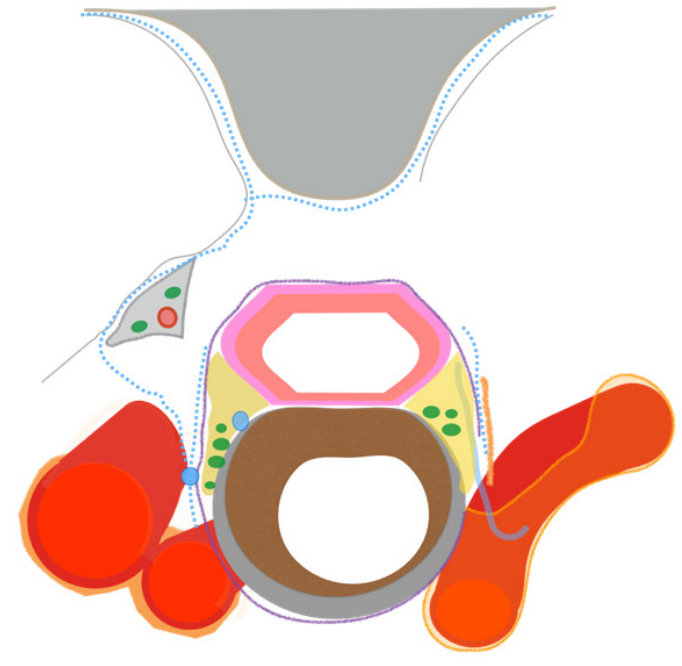

Figure 1. A: the microanatomical concept in the upper mediastinum; B: detaching the meso-esophagus while preserving the visceral sheath

\section{METHODS}

\section{Patients}

From June 2011 to January 2019, TEPP was performed in 500 patients (430 males, 70 females) with esophageal cancer at Okayama University Hospital. After reaching about 350 cases in April 2017, we started the microanatomy-based standardization of UMLND using a magnified view through a $4 \mathrm{~K}$ ultraHD system, and established it after reaching about 400 cases in November 2017. In this study, two groups were compared for the analysis: a pre-standardization group (100 cases up to completing 350 cases) and a post-standardization group (100 cases after completing 400 cases), as in our previous study ${ }^{[19]}$. Cases with tumors invading surrounding organs (T4), with omission of UMLND, after thoracotomy, and cases of robotic surgery were excluded. Final analysis thus included 91 cases of the pre-standardization group, and 83 paired cases of the post-standardization group. This study was approved by the Ethics Committee of Okayama University Hospital (1811-009).

\section{Procedure of TEPP}

Positioning of the patient, placement of the thoracoscope and ports, and the basic procedure of TEPP were performed as previously reported ${ }^{[1,20]}$. Since April 2017, a 4 K ultra-HD camera system (IMAGE1 STM, Karl Storz, Tuttlingen, Germany and Visera 4K UHD, Olympus Corporation, Tokyo, Japan) has been used.

\section{Microanatomy-based standardization of left upper mediastinal lymph node dissection}

In our procedure, the concepts of the meso-esophagus and visceral sheath are important. The mesoesophagus contains the lymph nodes around the recurrent laryngeal nerve, and the visceral sheath wraps the esophagus, trachea, and bilateral meso-esophagus [Figure $1 \mathrm{~A}]^{[19]}$. First, we peel off the dorsal and lateral sides of the esophagus, preserving the visceral sheath. On the lateral side, adhesions around the visceral sheath are so tight that we always have to peel it off together with branches of the sympathetic nerve [Figure $1 \mathrm{~B}$ and $3 \mathrm{~A}$ ]. Furthermore, on the right side, we also peel it off together with the vascular sheath [Figure 1B]. Next, we detach the esophagus and the meso-esophagus from the trachea and aggregate the lymphatic chain to the esophageal side [Figures $2 \mathrm{~A}, \mathrm{D}$ and $3 \mathrm{~B}$ ]. Next, we proceed with lymph node dissection along the recurrent laryngeal nerve from the central to the peripheral part. On the left side especially, we flip up the 
A

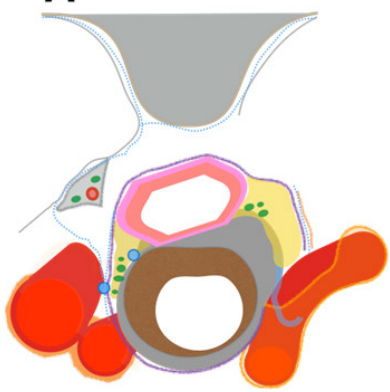

D

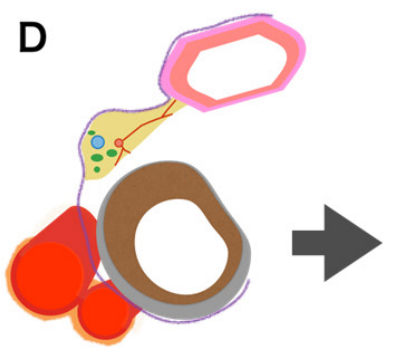

B

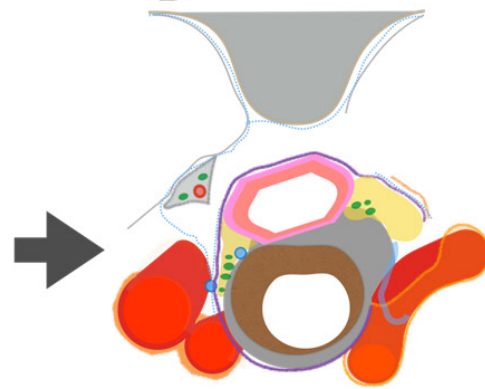

$\mathrm{E}$

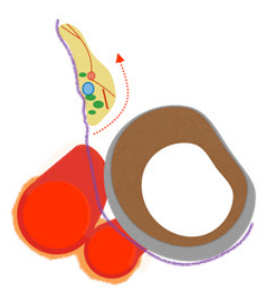

C

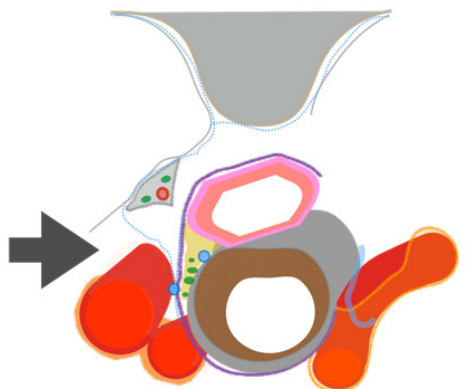

G

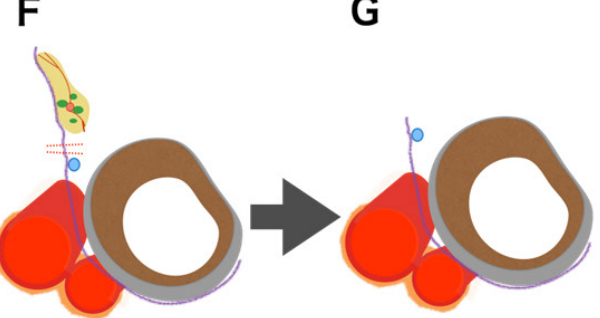

Figure 2. Microanatomy-based standardization of upper mediastinal lymph node dissection. A, D: detaching the esophagus together with the lymphatic chain from the trachea and aggregating the lymphatic chain to the esophageal side; $B, E$, F: identifying the recurrent laryngeal nerve and lymph node dissection along the nerve from the central part to the peripheral part; C, G: final findings of upper mediastinal lymph node dissection
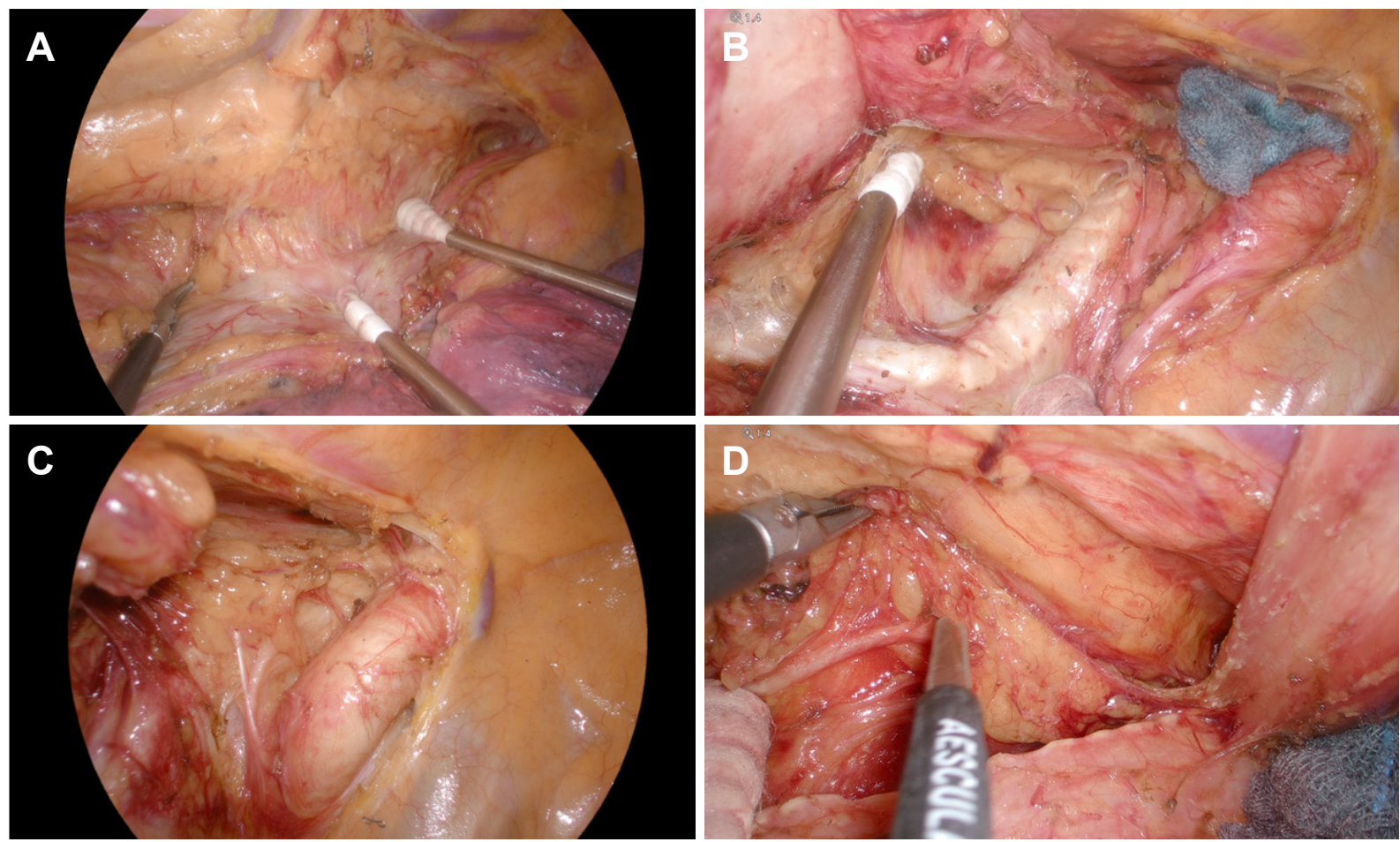

Figure 3. Thoracoscopic $4 \mathrm{~K}$ ultra-high-definition view. A: detaching the meso-esophagus on the left side while preserving the visceral sheath; B: aggregating the lymphatic chain to the esophageal side on the left side; C: upper mediastinal lymph node dissection on the right side; D: upper mediastinal lymph node dissection on the left side

lymphatic chain on the inner surface of the visceral sheath and slide the nerve down to the natural position [Figures 2B, E, F and 3C, D]. Finally, we cut the visceral sheath on the dorsal side of the nerve [Figure $2 \mathrm{C}, \mathrm{G}$ ]. 
Table 1. Patients' characteristics

\begin{tabular}{|c|c|c|c|}
\hline \multirow{2}{*}{ Characteristics } & \multicolumn{2}{|c|}{ Total cohort } & \multirow{2}{*}{$P$ value } \\
\hline & Pre-standardization group $(n=91)$ & Post-standardization group $(n=83)$ & \\
\hline Age, median [years (IQR)] & $67(61-72)$ & $67(60-73)$ & $0.744^{\mathrm{a}}$ \\
\hline \multicolumn{4}{|l|}{ Sex } \\
\hline Male (\%) & $75(82.4)$ & $68(81.9)$ & $0.933^{b}$ \\
\hline Female (\%) & $16(17.6)$ & $15(18.1)$ & \\
\hline $\mathrm{BMI}$, median $\left[\mathrm{kg} / \mathrm{m}^{2}(\mathrm{IQR})\right]$ & $21.3(19.6-23.2)$ & $22.1(20.3-23.5)$ & $0.216^{\mathrm{a}}$ \\
\hline Neoadjuvant chemotherapy (\%) & $53(58.2)$ & $45(54.2)$ & $0.592^{b}$ \\
\hline \multicolumn{4}{|l|}{ Clinical stage (UICC 8th) } \\
\hline O, I, II (\%) & $55(60.4)$ & $51(61.4)$ & $0.892^{b}$ \\
\hline III, IV (\%) & $36(39.6)$ & $32(38.6)$ & \\
\hline \multicolumn{4}{|l|}{ ASA-PS } \\
\hline $1(\%)$ & $22(24.2)$ & $24(28.9)$ & $0.414^{b}$ \\
\hline $2(\%)$ & $49(53.8)$ & $47(56.6)$ & \\
\hline $3(\%)$ & $20(22.0)$ & $12(14.5)$ & \\
\hline \multicolumn{4}{|l|}{ Tumor location } \\
\hline $\mathrm{Ce}(\%)$ & $5(5.5)$ & $6(7.2)$ & $0.292^{b}$ \\
\hline Ut (\%) & $9(9.9)$ & $17(20.5)$ & \\
\hline Mt (\%) & $46(50.5)$ & $36(43.4)$ & \\
\hline Lt (\%) & $23(25.3)$ & $20(24.1)$ & \\
\hline $\mathrm{Ae}(\%)$ & $8(8.8)$ & $4(4.8)$ & \\
\hline \multicolumn{4}{|l|}{ Histological diagnosis } \\
\hline $\operatorname{SCC}(\%)$ & $82(90.1)$ & $76(91.6)$ & $0.099^{b}$ \\
\hline $\operatorname{ADC}(\%)$ & $6(6.6)$ & $4(4.8)$ & \\
\hline Others (\%) & $3(3.3)$ & $3(3.6)$ & \\
\hline \multicolumn{4}{|l|}{ Lymph node dissection } \\
\hline Two-field dissection (\%) & $49(53.8)$ & $34(41.0)$ & $0.089^{b}$ \\
\hline Three-field dissection (\%) & $42(46.2)$ & $49(59.0)$ & \\
\hline \multicolumn{4}{|l|}{ Operator experience } \\
\hline$\geq 20$ years $(\%)$ & $61(67.0)$ & $22(26.5)$ & $<0.001^{b}$ \\
\hline$<20$ years $(\%)$ & $30(33.0)$ & $61(73.5)$ & \\
\hline
\end{tabular}

${ }^{a}$ Mann-Whitney test, ${ }^{b} \chi^{2}$ test. IQR: interquartile range; BMI: body mass index; Ce: cervical esophagus; Ut: upper thoracic esophagus; Mt: middle thoracic esophagus; Lt: lower thoracic esophagus; Ae: abdominal esophagus; SCC: squamous cell carcinoma; ADC: adenocarcinoma

\section{Description and statistical analysis}

Clinicopathological factors were noted according to the Japanese Classification of Esophageal Cancer ${ }^{[21,22]}$ and the Union for International Cancer Control TNM Classification of Malignant Tumors, 8th edition ${ }^{[23]}$. Postoperative complications were categorized using the Clavien-Dindo classification ${ }^{[24]}$ [Table 1]. To evaluate differences between the two groups, continuous variables were assessed using the MannWhitney test, and categorical variables were assessed using the $\chi^{2}$ test or Fisher's exact test. Differences were considered significant when $P$ values were $<0.05$. All analyses were performed using JMP version 14 statistical analysis software (SAS Institute, Cary, NC, USA). The thoracoscopic operative time learning curve was analyzed using the moving average method ${ }^{[25,26]}$. With the moving average method, using the mean of thoracoscopic operative times, the trends are clarified and the changes are smoothened. A 20-case moving average was used in this study, and the exclusion criteria of the cases were the same as above.

\section{RESULTS}

\section{Patients' characteristics}

There were no significant differences in patient characteristics between the two groups [Table 1]. On the other hand, the rate of surgeries performed by operators with less than 20 years' experience was significantly higher in the post-standardization group $(P<0.001)$. 
Table 2. Surgical findings

\begin{tabular}{|c|c|c|c|}
\hline Variable & Pre-standardization group $(n=91)$ & Post-standardization group $(n=83)$ & $P$ value \\
\hline \multicolumn{4}{|l|}{ Intraoperative findings } \\
\hline Thoracoscopic operative time $[\min (I Q R)]$ & $232.0(202.8-264.0)$ & $209.0(176.0-235.0)$ & $<0.001^{2}$ \\
\hline Blood loss $[\mathrm{mL}(\mathrm{IQR})]$ & $200(100-330)$ & $200(105-400)$ & $0.764^{\mathrm{a}}$ \\
\hline Number of dissected No. 106 lymph nodes (IQR) & $11(8-15)$ & $10(8-13)$ & $0.137^{\mathrm{a}}$ \\
\hline Conversion to thoracotomy (\%) & $0(0)$ & $0(0)$ & $1.000^{b}$ \\
\hline \multicolumn{4}{|l|}{ Postoperative findings } \\
\hline Total morbidity $[\geq$ Grade II (\%)] & $48(52.7)$ & $37(44.6)$ & $0.250^{b}$ \\
\hline Respiratory complications [ $\geq$ Grade II (\%)] & $15(16.3)$ & $14(16.9)$ & $0.946^{b}$ \\
\hline Recurrent laryngeal nerve palsy $[\geq$ Grade I (\%)] & $18(19.8)$ & $8(9.6)$ & $0.061^{b}$ \\
\hline Anastomotic leakage $[\geq$ Grade II (\%)] & $7(7.7)$ & $9(10.8)$ & $0.472^{b}$ \\
\hline ICU stay [days (IQR)] & $6(5-7)$ & $6(5-6)$ & $0.742^{\mathrm{a}}$ \\
\hline Postoperative hospital stay [days (IQR)] & $21(17-26)$ & $22(17-27)$ & $0.782^{a}$ \\
\hline In-hospital mortality (\%) & $0(0)$ & $0(0)$ & $1.000^{\mathrm{b}}$ \\
\hline
\end{tabular}

${ }^{a}$ Mann-Whitney test, ${ }^{b} \chi^{2}$ test. Complications are described according to the Clavien-Dindo classification ${ }^{[24]}$. IQR: interquartile range; ICU: intensive care unit

\section{Clinical outcomes}

There were no significant differences between the two groups in the amount of blood loss during surgery, and the number of dissected lymph nodes around the recurrent laryngeal nerves (No. 106). In both groups, no patients required conversion to open thoracotomy [Table 2]. There were no significant differences in total morbidity rate, the incidence of respiratory complications or anastomotic leakage ( $\geq$ Grade 2 ). Regarding RLN palsy, vocal cord motility was checked in all patients by endoscopy on postoperative day 1 , and any dysmotility was defined as RLN palsy ( $\geq$ Grade 1 ). The incidence of RLN palsy decreased to less than half $(19.8 \%$ to $9.6 \%)$ after standardization $(P=0.061)$ [Table 2]. There were also no significant differences in ICU stay and postoperative hospital stay, and there were no postoperative mortalities in either group.

\section{Change in thoracoscopic operative time}

There was a significant difference in thoracoscopic operative time between the pre-standardization group and the post-standardization group $[n=91,232.0(202.8-264.0) \min v s . n=83,209.0$ (176.0-235.0) $\mathrm{min}$, $(P<0.001)$ ] [Table 2]. The moving average curve showed that the thoracoscopic operative time decreased markedly during the phase of microanatomy-based standardization of UMLD (from 350 cases to 400 cases) and stabilized [Figure 4].

\section{DISCUSSION}

When most MIEs were performed via TELP, there were reports that precise mediastinal lymph node dissection by MIE was as feasible as that by open thoracotomy with the added advantages of lesser decrease in respiratory function and lower respiratory complication rates ${ }^{[4,5]}$. However, even now, it is important in TELP to have a special team composed of three experts (i.e., surgeon, assistant, and endoscopist) to perform the procedure smoothly. On the other hand, an excellent surgical field contributed simply by gravity and artificial pneumothorax without the need for an assistant is one advantage of TEPP. Furthermore, the improved ergonomics for the surgeon in TEPP is another advantage.

In the early phase of introducing TEPP in this decade, there were some reports about its conferred advantages for lymph node dissection, especially in the upper mediastinum ${ }^{[9-12]}$. Along with the recent, remarkable progress of endoscopic optical instruments such as $3 \mathrm{D}$ and $4 \mathrm{~K}$ ultra-HD, there have also been reports about the microanatomy-based surgical concept for MIE, similar to total meso-rectal excision in rectal cancer surgery ${ }^{[13-18]}$. More recently, we have established the microanatomy-based standardization using the concept of the meso-esophagus wrapped with the visceral and vascular sheaths and reported 


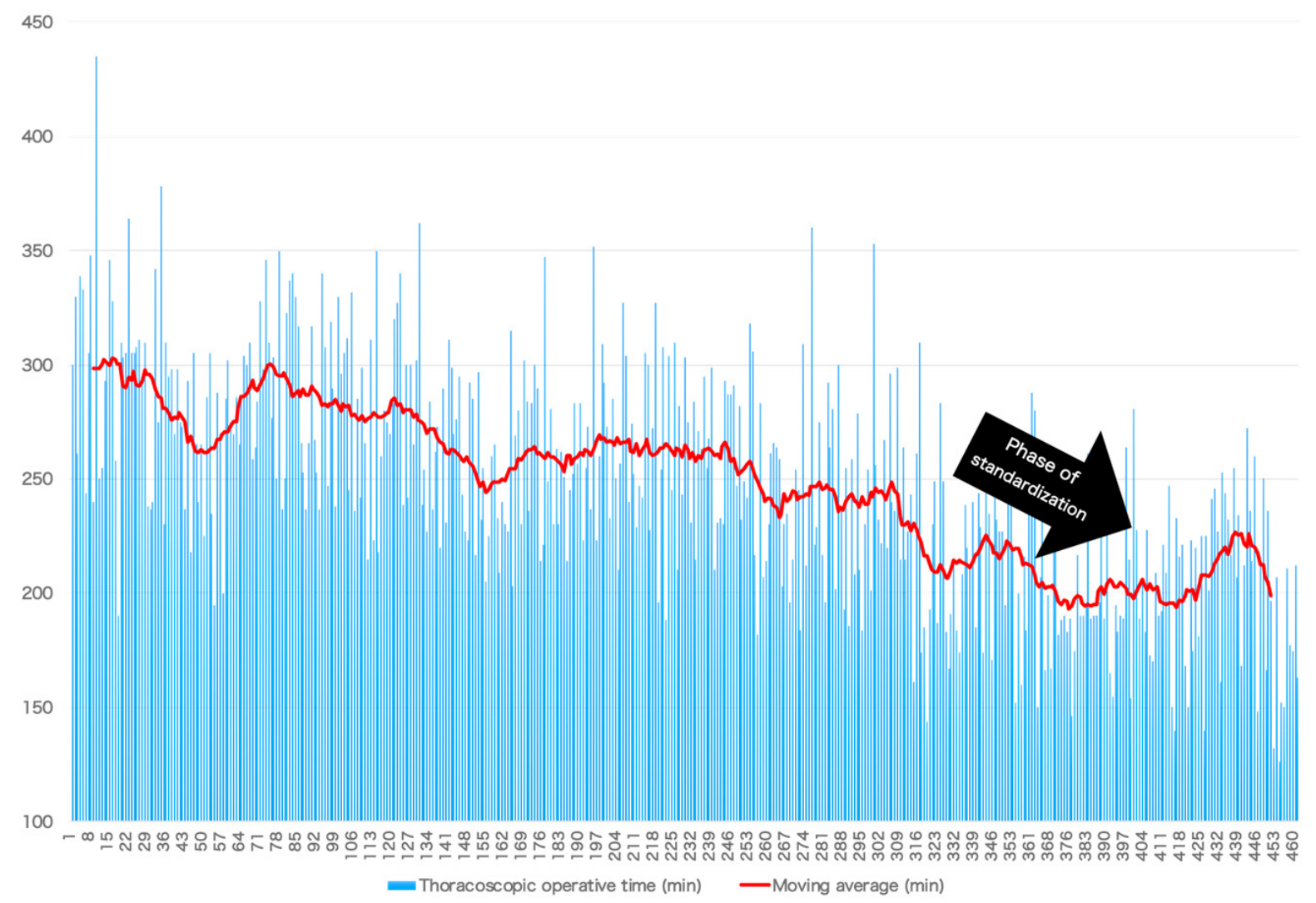

Figure 4. Twenty-case moving average of thoracoscopic operative time

its usefulness for left UMLND ${ }^{[19]}$. Although we thought that this concept could also be applied for right UMLND, the asymmetrical anatomical structure in the upper mediastinum was an issue. The branching patterns of the arteries are different and the points of recurrence of the recurrent laryngeal nerves and their running direction and length are also different. However, for both the right and left sides, the recurrent laryngeal nerves originate from each main trunk of the vagal nerve within the vascular sheath and transit to the inner aspect of the visceral sheath after turning back at an artery. The nerves then run to the larynx as their target organ through the meso-esophagus. In addition, lymph nodes around the recurrent laryngeal nerve that should be dissected as regional lymph nodes are located within the meso-esophagus on both sides. In the first step of our standardization of UMLND, it is most important not to destroy the visceral sheath enveloping the lymphatic chain in the meso-esophagus, although the vascular sheath on each side should be detached in a different manner and range. After that, we proceed to en bloc lymph node dissection while preserving the visceral sheath and nerve.

Regarding thoracoscopic operative time, the first learning curve occurred due to the initial standardization of the lower mediastinal procedure of $\mathrm{TEPP}^{[20]}$. After the initial standardization, the indication was expanded for thoracoscopic surgery. The thoracoscopic operative time again lengthened, followed by a second gentle, natural learning curve. However, after that, no obvious learning curve effect was achieved until more than 300 cases. Nevertheless, the microanatomy-based standardization during this time from case 350 to 400 contributed to a marked decrease in thoracoscopic operative time (by almost $30 \mathrm{~min}$ ), even though the number of surgeries performed by less experienced operators increased. We believe that accurate understanding of the microanatomy involved contributed towards defining the surgical planes more easily for quick dissection of tissues including the lymph nodes with without extra bleeding. On the other hand, young surgeons could also learn the microanatomy from experienced surgeons through 
the clear $4 \mathrm{~K}$ HD images and could re-watch the operation many times. Furthermore, there was also a concurrent decrease in postoperative complication rates. Therefore, our microanatomy-based concept appears to be useful not only for accurate and quick UMLD, but also for young surgeons to master the procedure efficiently.

Now, in our institution, we can conduct three types of MIE [thoracoscopic surgery, mediastinoscopic surgery, and robotic-assisted surgery (RAMIE)], and the rate of RAMIE has recently been increasing. We believe that RAMIE is an advanced form of thoracoscopic surgery and our microanatomy-based standardization approach could be similarly applied. Furthermore, we expect that this standardization will make much progress using the joint function and shake reduction system unique to RAMIE.

In conclusion, microanatomy-based standardization using a $4 \mathrm{~K}$ ultra-HD system enabled quicker and more precise UMLD despite an increase in the number of surgeries performed by less experienced operators.

\section{DECLARATIONS}

\section{Acknowledgments}

The authors would like to thank FORTE (https://www.forte-science.co.jp/) for English language review.

\section{Authors' contributions}

Conception and design: Shirakawa Y

Data collection: Shirakawa Y, Maeda N, Tanabe S

Establishment of study materials or enrolment of patients: Shirakawa Y, Noma K, Tanabe S, Sakurama K, Fujiwara T

Data scrutiny, analysis, and clarification: Shirakawa $Y$

Writing and revision of the manuscript: Shirakawa $Y$

Study supervision: Fujiwara T

Read and agree with the final manuscript: Shirakawa Y, Noma K, Maeda N, Tanabe S, Sakurama K, Fujiwara T

\section{Availability of data and materials}

Not applicable.

\section{Financial support and sponsorship}

None.

\section{Conflicts of interest}

All authors declared that there are no conflicts of interest.

\section{Ethical approval and consent to participate}

Not applicable.

\section{Consent for publication}

Not applicable.

\section{Copyright}

(c) The Author(s) 2020.

\section{REFERENCES}

1. Parkin DM, Bray F, Ferlay J, Pisani P. Global cancer statistics, 2002. CA Cancer J Clin 2005;55:74-108.

2. Akiyama H, Tsurumaru M, Kawamura T, Ono Y. Principles of surgical treatment for carcinoma of the esophagus: analysis of lymph node 
involvement. Ann Surg 1981;194:438-46.

3. Cuschieri A, Shimi S, Banting S. Endoscopic oesophagectomy through a right thoracoscopic approach. J R Coll Surg Edinb 1992;37:7-11.

4. Akaishi T, Kaneda I, Higuchi N, Kuriya Y, Kuramoto J, et al. Thoracoscopic en bloc total esophagectomy with radical mediastinal lymphadenectomy. J Thorac Cardiovasc Surg 1996;112:1533-40; discussion 40-1.

5. Osugi H, Takemura M, Higashino M, Takada N, Lee S, et al. A comparison of video-assisted thoracoscopic oesophagectomy and radical lymph node dissection for squamous cell cancer of the oesophagus with open operation. Br J Surg 2003;90:108-13.

6. Cuschieri A. Endoscopic subtotal oesophagectomy for cancer using the right thoracoscopic approach. Surg Oncol 1993;2:3-11.

7. Cuschieri A. Thoracoscopic subtotal oesophagectomy. Endosc Surg Allied Technol 1994;2:21-5.

8. Palanivelu C, Prakash A, Senthilkumar R, Senthilnathan P, Parthasarathi R, et al. Minimally invasive esophagectomy: thoracoscopic mobilization of the esophagus and mediastinal lymphadenectomy in prone position--experience of 130 patients. J Am Coll Surg 2006;203:7-16.

9. Noshiro H, Iwasaki H, Kobayashi K, Uchiyama A, Miyasaka Y, et al. Lymphadenectomy along the left recurrent laryngeal nerve by a minimally invasive esophagectomy in the prone position for thoracic esophageal cancer. Surg Endosc 2010;24:2965-73.

10. Ozawa S, Ito E, Kazuno A, Chino O, Nakui M, et al. Thoracoscopic esophagectomy while in a prone position for esophageal cancer: a preceding anterior approach method. Surg Endosc 2013;27:40-7.

11. Tsujimoto H, Takahata R, Nomura S, Yaguchi Y, Kumano I, et al. Video-assisted thoracoscopic surgery for esophageal cancer attenuates postoperative systemic responses and pulmonary complications. Surgery 2012;151:667-73.

12. Yatabe T, Kitagawa H, Yamashita K, Hanazaki K, Yokoyama M. Comparison of the perioperative outcome of esophagectomy by thoracoscopy in the prone position with that of thoracotomy in the lateral decubitus position. Surg Today 2013;43:386-91.

13. Akagawa S, Hosogi H, Yoshimura F, Kawada H, Kanaya S. Mesenteric excision for esophageal cancer surgery: based on the concept of mesotracheoesophagus. Int Cancer Conf J 2018;7:117-20.

14. Oshikiri T, Yasuda T, Harada H, Goto H, Oyama M, et al. A new method (the "Bascule method") for lymphadenectomy along the left recurrent laryngeal nerve during prone esophagectomy for esophageal cancer. Surg Endosc 2015;29:2442-50.

15. Cuesta MA, van der Wielen N, Weijs TJ, Bleys RL, Gisbertz SS, et al. Surgical anatomy of the supracarinal esophagus based on a minimally invasive approach: vascular and nervous anatomy and technical steps to resection and lymphadenectomy. Surg Endosc 2017;31:1863-70.

16. Cuesta MA, Weijs TJ, Bleys RL, van Hillegersberg R, van Berge Henegouwen MI, et al. A new concept of the anatomy of the thoracic oesophagus: the meso-oesophagus. Observational study during thoracoscopic esophagectomy. Surg Endosc 2015;29:2576-82.

17. Tokairin Y, Nakajima Y, Kawada K, Hoshino A, Okada T, et al. Histological study of the thin membranous structure made of dense connective tissue around the esophagus in the upper mediastinum. Esophagus 2018;15:272-80.

18. Fujiwara H, Kanamori J, Nakajima Y, Kawano T, Miura A, et al. An anatomical hypothesis: a "concentric-structured model" for the theoretical understanding of the surgical anatomy in the upper mediastinum required for esophagectomy with radical mediastinal lymph node dissection. Dis Esophagus 2019;32:doy119.

19. Shirakawa Y, Noma K, Maeda N, Tanabe S, Sakurama K, et al. Microanatomy-based standardization of left upper mediastinal lymph node dissection in thoracoscopic esophagectomy in the prone position. Surg Endosc 2020; epub ahead of print. doi: 10.1007/s00464-02007407-9

20. Shirakawa Y, Noma K, Maeda N, Katsube R, Tanabe S, et al. Assistant-based standardization of prone position thoracoscopic esophagectomy. Acta Med Okayama 2014;68:111-7.

21. Japan Esophageal Society. Japanese Classification of Esophageal Cancer, 11th Edition: part I. Esophagus 2017;14:1-36.

22. Japan Esophageal Society. Japanese Classification of Esophageal Cancer, 11th Edition: part II and III. Esophagus 2017;14:37-65.

23. Brierley JD, Gospodarowicz MK, Wittekind C. TNM classification of malignant tumors (UICC international union against cancer). 8th ed. Wiley-Blackwell, Oxford; 2017.

24. Dindo D, Demartines N, Clavien PA. Classification of surgical complications: a new proposal with evaluation in a cohort of 6336 patients and results of a survey. Ann Surg 2004;240:205-13.

25. Kayano H, Okuda J, Tanaka K, Kondo K, Tanigawa N. Evaluation of the learning curve in laparoscopic low anterior resection for rectal cancer. Surg Endosc 2011;25:2972-9.

26. Yamamoto M, Okuda J, Tanaka K, Kondo K, Asai K, et al. Evaluating the learning curve associated with laparoscopic left hemicolectomy for colon cancer. Am Surg 2013;79:366-71. 